\title{
Low cost Micro-Coulter counter with hydrodynamic focusing
}

\author{
R. Rodríguez-Trujillo, C. A. Mills, J. Samitier and G. Gomila \\ Laboratory of Nanobioengineering-CREBEC, Parc Cientific de Barcelona and \\ Departament of Electronics, University of Barcelona, C/ Marti i Franques, 1, \\ 08028-Barcelona, Spain \\ Phone: (+34) 934039147 \\ Fax: (+34) 934021148 \\ Email: romen@el.ub.es \\ URL: www.nanobiolab.pcb.ub.es
}

Article note: This research was supported by the Spanish Minister of Education and Science under project TEC2004-06514-C03-02

\begin{abstract}
We report on the fabrication and characterization of a low-cost micro-Coulter counter fabricated from biocompatible materials (Poly-dimethylsiloxane, glass and gold) and incorporating hydrodynamic focusing. The developed micro-Coulter counter offers a low-cost alternative to equivalent existing devices and, thanks to the hydrodynamic focusing, provides high versatility, being able to probe particles with a wide range of sizes within a single device. The device has been successfully tested for counting 20 latex micro beads in suspension.
\end{abstract}

Keywords: Micro-Coulter counter, hydrodynamic focusing, rapid prototyping, poly-dimethylsiloxane.

\section{Introduction}

The design and fabrication of microchips for cell and molecule handling has recently experienced a period of rapid development (Huh 2005; Erickson and Li 2004; Chován and Guttman 2002; Medoro et al 2003; Vilkner et al 2004). The aim of these developments is to develop particle counting, analysis and sorting systems to perform a variety of biotechnological applications on a chip. One particularly important application is the development of cell counters, which can be used as a stand alone device for counting, and sizing, or in more complex 
devices incorporating flow cytometry analysis for the discrimination and sorting of different cell populations (see Huh 2005 for a recent review on the subject).

Particle counting can be based on two detection methods. Optical methods extract information from light dispersed by particles and from specific fluorescent excitation (Schrum et al 1999; McClain et al 2001; Fu et al 2004), whereas electrical methods (referred to as Coulter counters) extract information from the modulation of the electrical resistance in an orifice due to the passage of the particle (Larsen et al 1997; Koch et al 1999; Ayliffe et al 1999; Saleh and Sohn 2001; Saleh and Sohn 2003; Saleh and Sohn 2003). Optical methods have the advantage of being able to incorporate fluorescent markers that are widely used in biology, but at the price of increased integration complexity. Electrical methods, on the other hand, present advantages from the point of view of integration, particularly after the demonstration of entirely electrical-based micro-flow cytometers and sorters utilizing electrical impedance methods (Cheung et al 2005; Holmes et al 2005; Morgan et al 2005).

Micro-Coulter counters, i.e. micro-dimensioned particle counters based on electrical detection methods, have been fabricated using a variety of materials -e. g. silicon (Schrum et al 1999; McClain et al 2001; Fu et al 2004; Larsen et al 1997; Koch et al 1999), glass (Ayliffe et al 1999), glass-polyimide (Cheung et al 2005; Holmes et al 2005; Morgan et al 2005; Gawad et al 2001), polymer (Saleh and Sohn 2003; Saleh and Sohn 2003), and quartz (Saleh and Sohn 2001)- and with different particle alignment methods -geometric (Saleh and Sohn 2001; Saleh and Sohn 2003; Saleh and Sohn 2003), hydrodynamic (Larsen et al 1997) and electrokinetic (Schrum et al 1999; McClain et al 2001; Fu et al 2004; Holmes et al 2005; Morgan et al 2005; Cheung et al 2005). Particles with diameters from several micrometers down to tens of nanometers (Saleh and Sohn 2001; Saleh and Sohn 2003; Saleh and Sohn 2003) have been examined with detection ratios varying from a few counts per second, if electrophoretic forces are utilized for particle manipulation (Saleh and Sohn 2001), to hundreds of counts per second, if pressure forces are utilized (Morgan et al 2005; Gawad et al 2001). Recent advances (Holmes et al 2005; Morgan et al 2005) suggest that pressure driven particles combined with dielectrophoretic alignment and impedance detection 
methods could provide the appropriate technology for high performance microflow cytometry. However, for various other applications and testing purposes a versatile low-cost alternative to these micro-Coulter counters might be desirable. Here we describe the development of one such alternative.

The micro-Coulter counter presented here consists of a pressure driven particle counter, with hydrodynamic focusing for particle alignment, and impedance detection for particle counting. The use of hydrodynamic focusing together with electrical detection (to our knowledge only previously reported in Larsen et al 1997) makes the device highly versatile, allowing the possibility of examining particles with a broad range of sizes in a single device. Fabrication of the channel structure of the device in a polymer (Poly-dimethylsiloxane (PDMS)) using a rapid prototyping technology (Qin et al 1996; McDonald et al 2000) offers low fabrication costs compared to equivalent devices based on micromachined silicon (Schrum et al 1999; McClain et al 2001; Fu et al 2004; Larsen et al 1997; Koch et al 1999) or microfabricated glass/pyrex (Cheung et al 2005; Holmes et al 2005).

\section{Device description}

A diagram of the layout of the micro-Coulter counter is shown in figure 1. It has three inlet reservoirs connected to their corresponding channels and converging into a single outlet channel leading to the outlet reservoir. All the channels are $\sim 190 \mu \mathrm{m}$ wide and all the reservoirs are $3 \mathrm{~mm}$ in diameter. The channels are all $\sim 50 \mu \mathrm{m}$ deep. Channel structures are fabricated in PDMS and sealed onto a glass substrate containing the microfabricated Ti-Au electrodes. The microelectrodes are coplanar and transversal to the direction of the flux. They are $40 \mu \mathrm{m}$ wide, 70 $\mathrm{nm}$ tall and have a $40 \mu \mathrm{m}$ separation. The detection zone is the volume located between the electrodes.

\section{Device fabrication}

Fabrication of the PDMS micro-channels has been performed by means of rapid prototyping (McDonald et al 2000). First, negative masks of the design presented 
in figure 1a are printed on transparent acetate sheets using an Agfa Selectset Avantra $44 E$ printer with a resolution of 3600 dpi. Then, this mask is used to expose a $50 \mu \mathrm{m}$ thin film of a negative photoresist ( $S U-8$ 50, MicroChem Corp., $U S A)$ previously spun and cured on a glass substrate. The resin is then developed (SU8 developer, Microchem Corp., USA) to obtain a negative mould of our required structure. Replication of the mould was achieved using a silicon-based elastomeric polymer, PDMS (Slygard 184, Microchem Corp., USA), by means of standard soft lithography techniques (Qin et al 1996). To prevent adhesion to the polymer in the replication process, a fluorosilane monolayer is added to the SU-8 master from the vapour phase, which acts as anti-sticking layer.

An array of 8 interdigited Ti-Au electrodes $40 \mu \mathrm{m}$ wide, $90 \mathrm{~nm}$ tall and with a 40 $\mu \mathrm{m}$ separation has been microfabricated on a glass substrate $(50 \mathrm{~mm} \times 70 \mathrm{~mm})$ by means of standard photolithography techniques.

The polymeric microchannels are sealed to the glass substrate containing the microelectrodes by exposure to oxygen plasma. This process is irreversible and, due to the ability of the PDMS to form conformal contact with a surface, the electrodes are incorporated between the PDMS and the glass. Figure 2 presents an optical image of the electrode region in the sealed structure.

Finally, external fluidic connections (Upchurch Scientific) are sealed on the glass surface over holes that were previously drilled to be coincident with the inlets and outlet of the PDMS channels. Copper wires are fixed to the external electrodes pads with a silver conductive paint ( $R S$ Amidata). For the experiments presented here only two of the 8 electrodes are probed.

The completed device with both electrical and fluidic connexions present is shown in figure 3. 


\section{Device characterization}

\subsection{Fluidic characterization}

A Harvard Apparatus PHD2000 Syringe Pump was utilised to inject a constant fluid flux during the experiment. Optical characterisation was made by means of a Photonfocus MV-D640C-33 colour camera connected to a Zeiss Axio Imager.A1m optical microscope.

Nine different fluid focalisation conditions were set by fixing the flux rate in the lateral inlet channels $\left(\mathrm{Q}_{\mathrm{f} 1}=\mathrm{Q}_{\mathrm{f} 2}=10 \mu \mathrm{l} / \mathrm{min}\right)$ and varying the flux rate in the central inlet channel $\left(\mathrm{Q}_{\mathrm{i}}\right)$ between $0,1 \mu \mathrm{l} / \mathrm{min}$ and $20 \mu \mathrm{l} / \mathrm{min}$. All channels were filled with deionised water (miliQ water by Millipore) and, in order to distinguish the limits between the three fluxes, a water based ink was utilised to dye the fluid inserted into the central channel. For every flux the width of the central flux $\left(\mathrm{w}_{0}\right)$ was measured. Figure 4 shows optical microscope images of the focalization region for four different focalisation conditions. The results for the width $\mathrm{w}_{0}$ of the central channel versus the flux rate $Q_{i}$ of the lateral channels are presented in figure 5 .

The experimental results are in complete agreement with the expected theoretical relation for this structure (the dashed line in figure 5) as (Stiles et al 2005):

$$
w_{0}=w \frac{Q_{i}}{Q_{i}+Q_{F}}
$$

where $w$ is the width of the PDMS channel $(189,78 \mu \mathrm{m}$ directly measured from an optical image $)$ and $\mathrm{Q}_{\mathrm{F}}$ is the total lateral flux $\left(\mathrm{Q}_{\mathrm{F}}=\mathrm{Q}_{\mathrm{f} 1}+\mathrm{Q}_{\mathrm{f} 2}\right)$.

The maximum fluid focalisation achieved with the present experimental set up corresponds to a central fluid width of about $2 \mu \mathrm{m}$. Given the height of the PDMS channel, $50 \mu \mathrm{m}$, the present device is expected to be useful for particles with diameters between $\sim 2 \mu \mathrm{m}$ and $\sim 50 \mu \mathrm{m}$. 


\subsection{Particle focalization}

The effect of hydrodynamic focusing was utilised to prove the correct alignment of particles in our device. $20 \mu \mathrm{m}$ diameter particles (polystyrenes particles from Polysciences) were suspended on water and made pass through the central channel of the micro-Coulter counter. The lateral channels were filled with D.I. water. Fluxes were adjusted using equation (1) in order to have a central channel width of $20 \mu \mathrm{m}$, making particles pass one by one. This process was verified with the help of the microscope and the camera. Figure 6 shows a single particle passing through the central channel in the focalised region of the device.

\subsection{Electrical characterization}

A Corning Conductivity meter 441 was utilised to measure the conductivities of the two solutions giving $4.42 \mu \mathrm{S} / \mathrm{cm}$ for the deionised water and $4200 \mu \mathrm{S} / \mathrm{cm}$ for the dyed, deionised water. Two neighbouring microelectrodes were excited with a $1 \mathrm{~V} \mathrm{AC}$ signal of $1 \mathrm{MHz}$ frequency and real time measurements of the impedance between the electrodes were made by means of an Agilent 4294A impedance analyser controlled with LabView 6.1.

Figure 7 presents the experimental results for the interelectrode resistance as a function of the width of the central flux when different focalisation conditions were set.

From the expression of the electrical resistance for a paralelepiped of a conductive material $(R=L /(\sigma h w)$; with $L$ being the length in the electrical current direction, $\sigma$ the conductivity of the medium and $h w$ the cross-sectional area of the paralelepiped) and considering the current flux to be homogeneous in the volume between the electrodes (the equivalent of considering the electrodes to be facing each other, perpendicular to the flux, instead of being coplanar) we can obtain the theoretical dependence of the resistance $R$ measured between two consecutive electrodes with the width of the central flux $w_{0}$. To this end one has to consider that the measured resistance is the parallel combination of two resistances: one 
corresponding to the central flow and another corresponding to the outside flows (which are assumed to have the same conductivity). One then has:

$$
R=\frac{1}{R_{\text {lateral }}^{-1}+R_{\text {central }}^{-1}}=\frac{\alpha}{\frac{h\left(w-w_{0}\right) \sigma}{L}+\frac{h}{L} \sigma^{\prime} w_{0}}
$$

where $\sigma$ and $\sigma$ ' are the conductivities of the deionised water and the dyed, deionised water respectively, $L$ is the distance between electrodes and $h$ is the height of the channel. In Eq. (2) $\alpha$ is a geometrical factor taking into account the effect of having coplanar electrodes instead of facing electrodes. This relation is presented in figure 7 (dashed line) and is in excellent agreement with the experimental data, when taking $\alpha=11.3$. The distance $L=113 \mu \mathrm{m}$ has been taken as the distance from the beginning of the first polarised electrode to the end of the second polarised electrode, directly measured from an optical microscope image (this would correspond to the inter-electrode distance in the approximation of electrodes facing one another).

Equations (1) and (2) prove that hydrodynamic focalization plays a double role in the present device: on the one hand it allows the particles to be aligned singly when passing over the measuring electrodes (Eq. 1), on the other hand, it allows the active area of the device to be effectively confined to the size of the focalized stream, thus enhancing the device sensitivity (Eq. 2) (Spielman and Goren 1968; Merkus et al 1990). This last property is simply achieved by using fluids with much higher conductivity in the central flow than in the lateral flows. Under such conditions the measured resistance is practically solely due to the central flux, whose width is comparable to the particle diameter, as dictated by the requirement for particle alignment.

This property is what gives great versatility to the developed device and allows it to be used for the counting of particles with a broad range of sizes within a single channel. 


\section{Particle counting}

In order to provide a test of the capabilities of the developed device, counting of latex micro beads in suspension has been performed. A suspension of latex micro beads in an aqueous solution of $\mathrm{NaCl}$ ions was prepared and introduced into the central channel of our device. The outside channels were filled with deionised water in order to confine the electrical current to the volume filled by the central channel. The conductivities of liquids were $10670 \mu \mathrm{S} / \mathrm{cm}$ for the central channel solution and $4.42 \mu \mathrm{S} / \mathrm{cm}$ for the lateral deionised water. The diameter of the particles was $20 \mu \mathrm{m}$. We have set the conductivity at $10670 \mu \mathrm{S} / \mathrm{cm}$ after optimization experiments to produce the best signal to noise ratio, while remaining inside the limits of a characteristic PBS solution.

Two neighbouring microelectrodes were excited with a $1 \mathrm{~V} \mathrm{AC}$ signal of $1 \mathrm{MHz}$ frequency and real time measures of the impedance between the electrodes were made. Focalization conditions where arranged so as to have a central channel width of $20 \mu \mathrm{m}$, thus guaranteeing the correct alignment of the latex micro beads and at the same time confining the active detection width to the width of the latex micro-beads.

The particles were monitored with the aid of an optical microscope in order to correlate particle passage and particle detection. To achieve this, low flow velocities where used. Each time a single particle passed the electrodes, a peak in the measured resistance was observed. Some characteristic peaks corresponding to the passage of a single $20 \mu \mathrm{m}$ diameter latex micro-bead are shown in figure 8 . A resistance variation of approximately $6 \%$ has been obtained with respect to the baseline. A relative noise ratio of $2 \%$ enables us to easily distinguish the transitions. Characteristic transition times are approximately $50 \mathrm{~ms}$, potentially allowing us to measure up to 20 particles per second. However, this was at a low flow velocity so that the passage of the beads could be optically monitored, higher flow velocities are expected to reduce the transition time and hence increase the counting rate. 
Finally, it is worth emphasising that the hydrodynamic focalization allows the detection of particles as small as $20 \mu \mathrm{m}$ in diameter in a device with a physical cross-section as large as $190 \times 50 \mu \mathrm{m}^{2}$.

\section{Conclusions}

In the present work we have reported on the design, fabrication and characterization of a low cost micro-Coulter counter made using biocompatible materials. The device incorporates hydrodynamic focalization designed to both increase device sensitivity and to analyze particles of different diameter in a single device. The present device could offer a valid low cost alternative to equivalent devices based on micromachined silicon or microfabricated glass/pyrex.

Acknowledgements:

We kindly acknowledge support from B. Sanahuja for the development of the Labview software and from M. Castellarnau for assistance in the impedance measurements. The device was fully fabricated within the Nanotechnology Platform facility of the Barcelona Science Park. We gratefully acknowledged the work performed by E. Martínez and M. J. López. 
References:

Ayliffe H E et al. (1999) Electric impedance spectroscopy using microchannels with integrated metal electrodes. IEEE J Microelectromech S 8:50-57. DOI: 10.1109/84.749402

Cheung K et al. (2005) Impedance spectroscopy flow cytometry: on-chip label-free cell differentiation. Cytom Part A 65A:124-132. DOI: 10.1002/cyto.a.20141

Chován T, Guttman A (2002) Microfabricated devices in biotechnology and biochemical processing. Trends Biotechnol 20: 116-122. DOI: 10.1016/S0167-7799(02)01905-4

Erickson D, Li D (2004) Integrated microfluidic devices. Anal Chim Acta 507: 11-26. DOI: 10.1016/j.aca.2003.09.019

Fu L-M et al. (2004) Electrokinetically driven microflow cytometers with integrated fiber optics for on-line cell/particle detection. Anal Chim Acta 507:163-169. DOI: 10.1016/j.aca.2003.10.028

Gawad S et al. (2001) Micromachined impedance spectroscopy flow cytometer for cell analysis and particle sizing. Lab Chip 1:76-82. DOI: 10.1039/b103933b

Holmes D et al. (2005) On-chip high-speed sorting of micron-sized particles for high-throughput analysis. IEE Proc.-Nanobiotechnol 152:129-135. DOI:10.1049/ip-nbt:20050008

Huh D et al. (2005) Microfluidics for flow cytometric analysis of cells and particles. Physiol Meas 26:R73-R98. DOI: 10.1088/0967-3334/26/3/R02

Koch M et al. (1999) Design and fabrication of a micromachined Coulter counter. J Micromech Microeng 9:159-161. DOI:10.1088/0960-1317/9/2/313

Larsen U D et al. (1997) Microchip coulter particle counter. Proceedings of the International Conference on Solid State Sensors and Actuators 1319-1322. DOI:

10.1109/SENSOR.1997.635479

McClain M A et al. (2001) Flow cytometer of Escherichia Coli on microfluidic devices. Anal Chem 73:5334-5338. DOI: 10.1021/ac010504v

McDonald J C et al. (2000) Fabrication of microfluidic systems in poly(dimethylsiloxane). Electrophoresis 21:27-40. DOI: 10.1002/(SICI)1522-2683(20000101)21:1<27::AIDELPS27>3.0.CO;2-C 
Medoro G et al. (2003) A Lab-On-A-Chip for cell detection and manipulation. IEEE Sens J 3:317325. DOI: 10.1109/JSEN.2003.814648

Merkus H G et al. (1990) Improved resolution and accuracy in electrical sensing zone particle counters through hydrodynamic focusing. Part Part Syst Char 7:11-15 DOI:

10.1002/ppsc. 19900070103

Morgan $\mathrm{H}$ et al. (2005) High speed simultaneous optical and impedance analysis of single particles. Curr Appl Phys (in press) DOI: 10.1016/j.cap.2005.11.020

Qin D et al. (1996) Rapid prototyping of complex structures with feature sizes larger than $20 \mu \mathrm{m}$. Adv Mater 8:917-919. DOI: 10.1002/adma.19960081110

Saleh O A, Sohn L L (2001) Quantitative sensing of nanoscale colloids using a microchip Coulter counter. Rev Sci Instrum 72:4449-4451. DOI: 10.1063/1.1419224

Saleh O A, Sohn L L (2003) Direct detection of antibody-antigen binding using an on-chip artificial pore. P Natl Acad Sci Usa 100:820-824. DOI: 10.1073/pnas.0337563100

Saleh O A, Sohn L L (2003) An artificial nanopore for molecular sensing. Nano Lett 3:37-38 DOI:10.1021/n10255202

Schrum D P et al. (1999) Microchip flow cytometry using electrokinetic focusing. Anal Chem 71:4173-4177. DOI: 10.1021/ac.990372u

Spielman L, Goren S L (1968) Improving resolution in Coulter counting by hydrodynamic focusing. J Coll Int Sci 26:175-182. DOI: 10.1016/0021-9797(68)90310-X

Stiles T et al. (2005) Hydrodynamic focusing for vacuum-pumped microfluidics. Microfluid Nanofluid 1:280-283. DOI: 10.1007/s10404-005-0033-z

Vilkner T et al. (2004) Micro Total Analysis Systems. Recent Developments. Anal Chem 76:33733386. DOI: $10.1021 / \mathrm{ac} 040063 \mathrm{q}$ 
Published in: Microfluidics and Nanofluidics 3:2 (2007) 171-176

Figure legends:

Figure 1: Design of the micro-Coulter counter structure. (a) Plan view and (b) longitudinal section of the central channel (dotted line in figure 1a). The diagrams are not to scale.

Figure 2: Optical microscope image of the detection zone. The gold electrodes can be distinguished running perpendicular to the channel. In the experiments only two consecutive electrodes were polarized.

Figure 3: Optical image of the final device structure incorporating external fluidic and electrical connections.

Figure 4: Optical microscope images showing the effect of the hydrodynamic focalization. The width of the channel w is $190 \mu \mathrm{m}$. $Q_{f 1}$ and $Q_{f 2}$ are always taken to be $10 \mu \mathrm{l} / \mathrm{min}$ and $Q_{i}$ is decreased causing $\mathrm{w}_{0}$ to be reduced: (a) $Q_{i}=20 \mu \mathrm{l} / \mathrm{min}, \mathrm{w}_{0}=90.4 \mu \mathrm{m}$ (b) $Q_{i}=5 \mu \mathrm{l} / \mathrm{min}, \mathrm{w}_{0}=37.52 \mu \mathrm{m}$ (c) $Q_{i}=1$ $\mu \mathrm{l} / \mathrm{min}, \mathrm{w}_{0}=11.94 \mu \mathrm{m}(\mathrm{d}) Q_{i}=0.1 \mu \mathrm{l} / \mathrm{min}, \mathrm{w}_{0}=2.32 \mu \mathrm{m}$

Figure 5: Reduction of the central flux width as a consequence of decreasing the flux rate in the central fluid flow (hydrodynamic focalization). The experimental results (dots) are compared to a theoretical curve (dashed line), as described in the text [Eq. (1)].

Figure 6: Optical microscope image showing a focalised particle passing through the central flux.

Figure 7: Resistance measured between two neighbouring electrodes as a function of the width of the central (dyed) flux $\left(\mathrm{w}_{0}\right)$. The experimental measurements (circles) can be compared to the expected behaviour derived from theory [Eq. 2] (dashed line). The cases where $\mathrm{w}_{0}=0$ and $\mathrm{w}_{0}=190 \mu \mathrm{m}$ correspond to filling the entire channel with deionised water and with dyed water respectively.

Figure 8: Time evolution of the resistance signal showing eight $20 \mu \mathrm{m}$ particle transitions. 
Figure 1

(a)

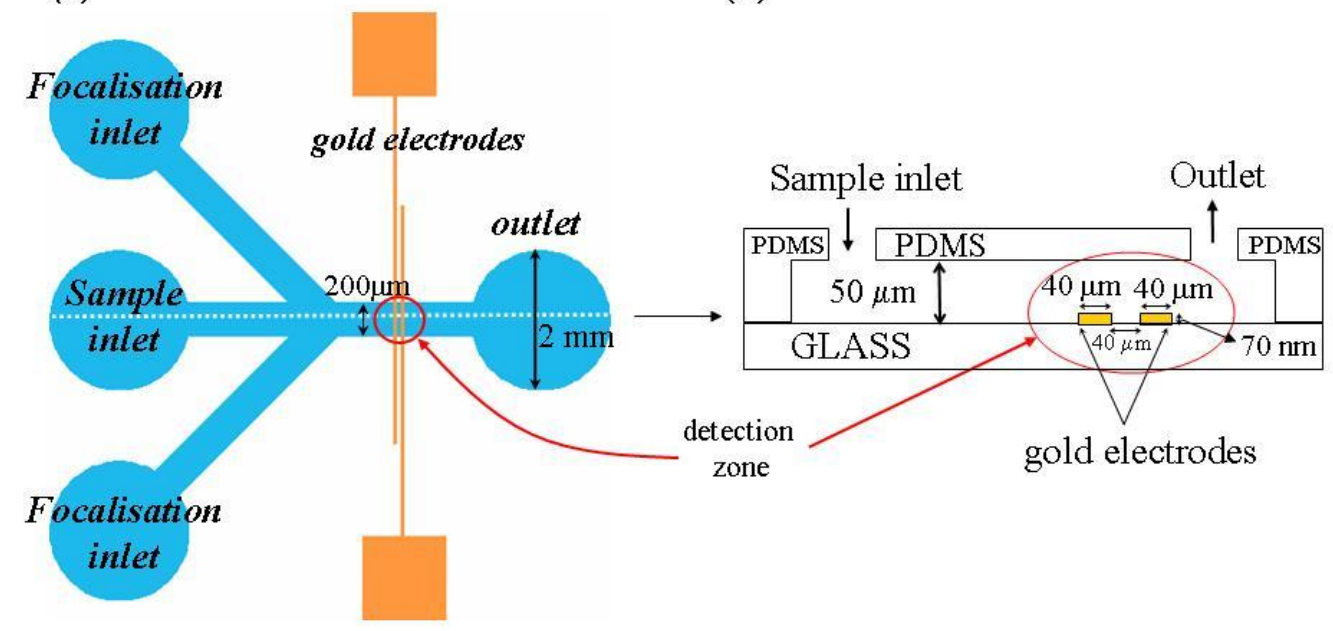

Figure 2

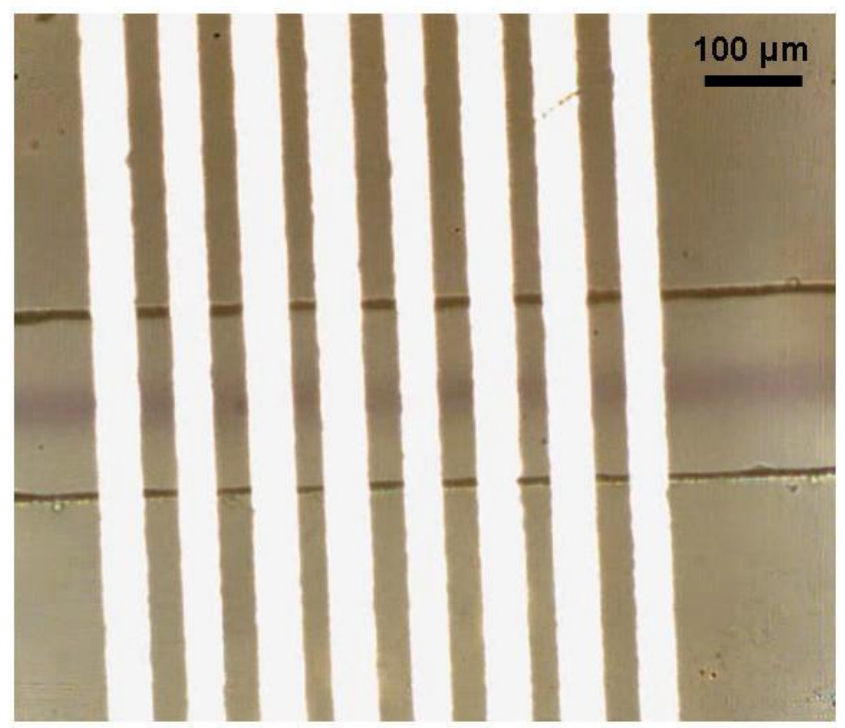


Published in: Microfluidics and Nanofluidics 3:2 (2007) 171-176

Figure 3

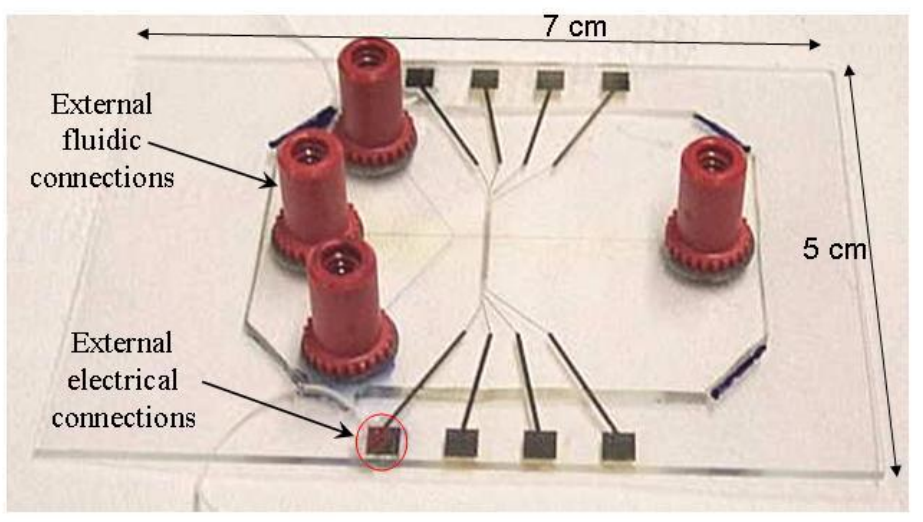

Figure 4

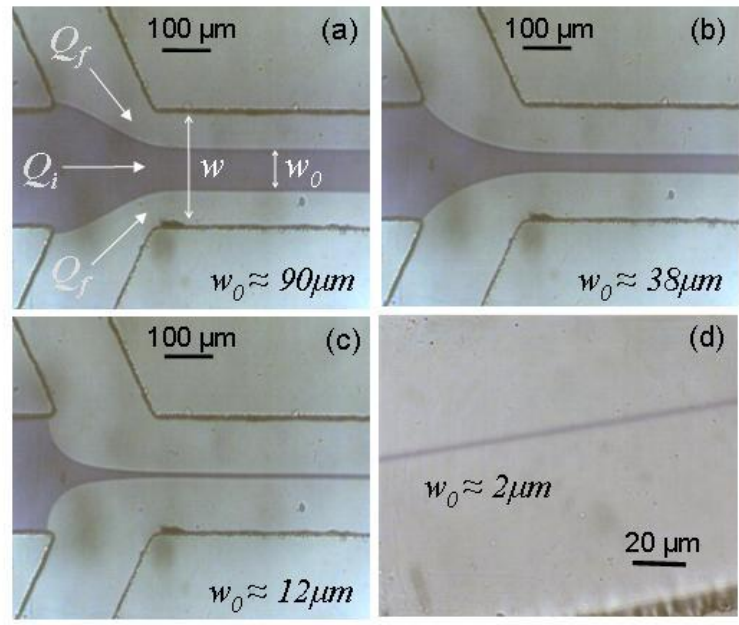


Published in: Microfluidics and Nanofluidics 3:2 (2007) 171-176

Figure 5

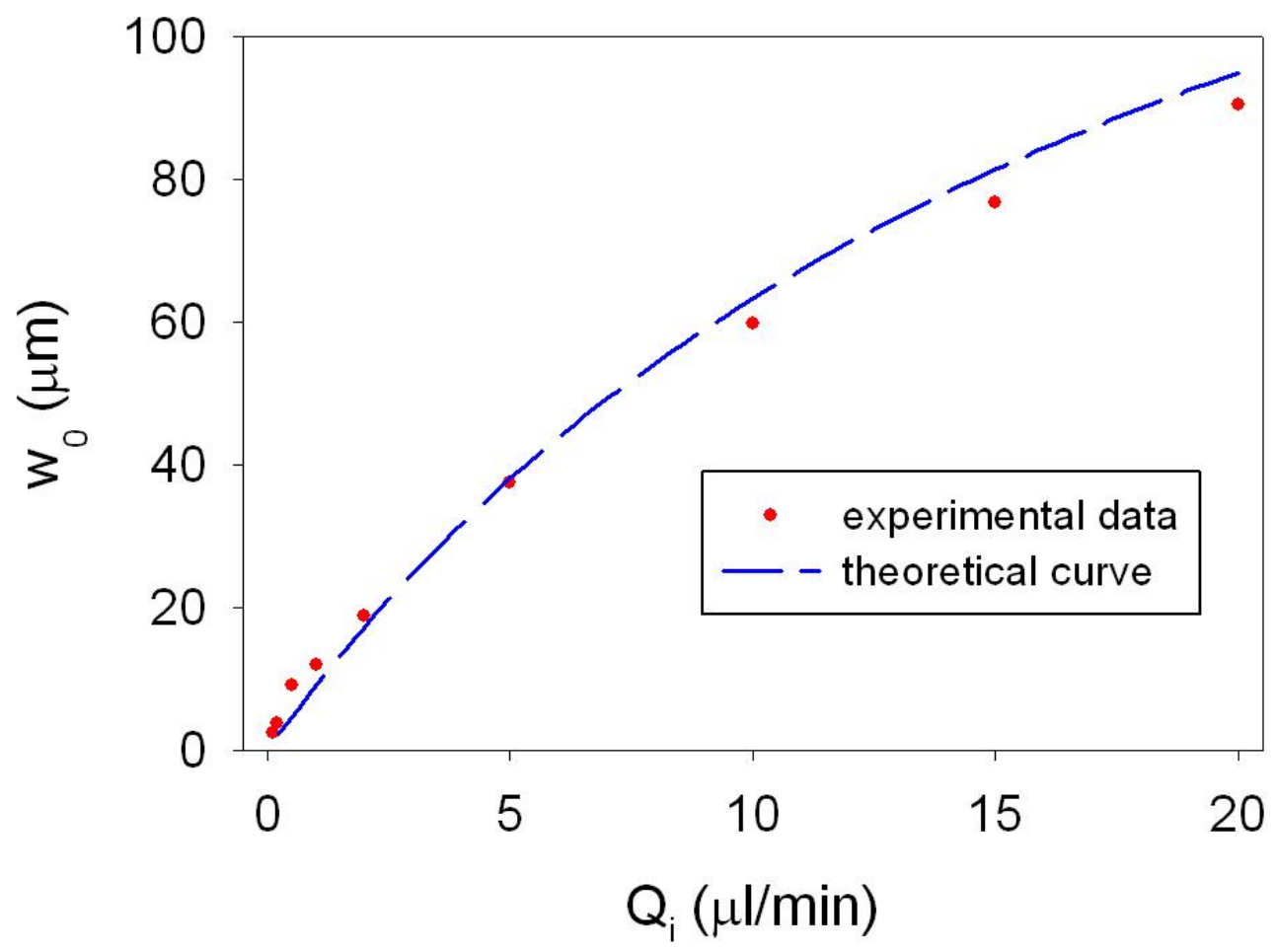

Figure 6 
Published in: Microfluidics and Nanofluidics 3:2 (2007) 171-176

Figure 7

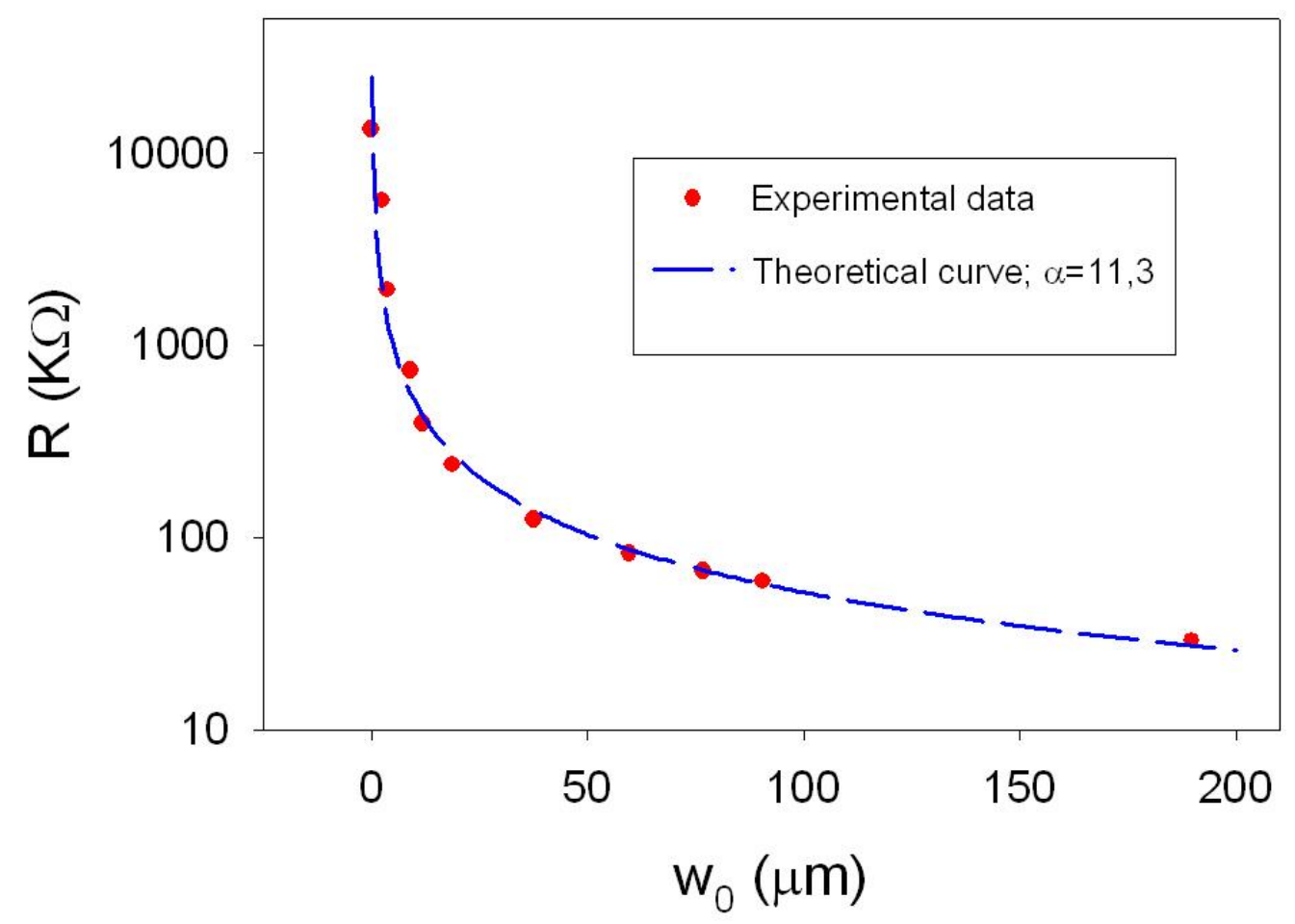

Figure 8

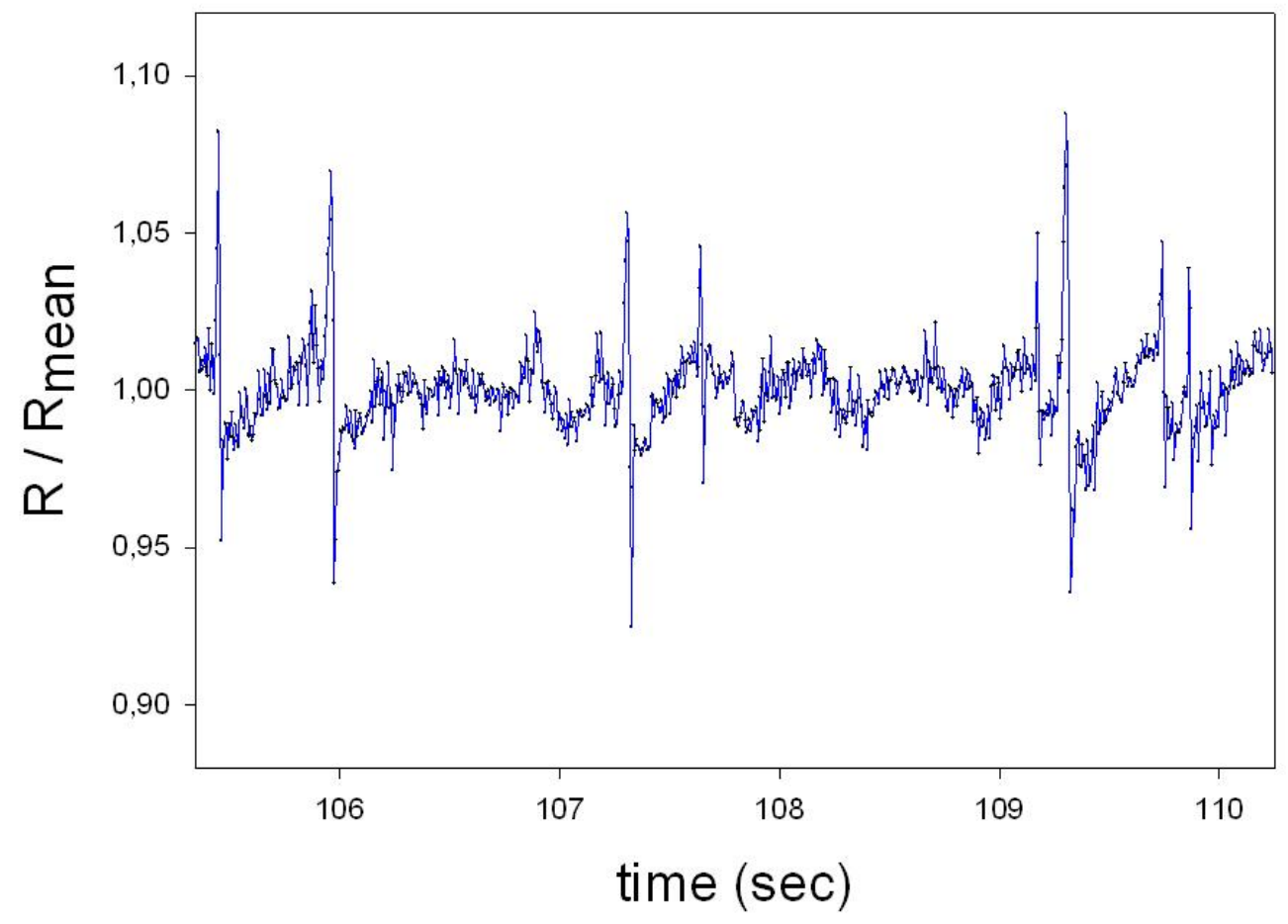

